Revista Digital Universitaria

Vol. 19, Núm. 4, julio-agosto 2018

\title{
¿Cuánto vale tu felicidad?
}

\author{
Gonzalo Soltero
}

\section{Resumen}

Este artículo revisa el alto grado de bienestar subjetivo de los mexicanos y en especial de sus estudiantes. Con el objetivo de dar cuenta del valor que los estudios tienen para nuestra felicidad, asigno una expresión monetaria tentativa a las décimas que integran nuestro promedio de bienestar subjetivo. Asimismo, concluyo con algunas consideraciones respecto a ciertos aspectos que podrían fomentar aún más los beneficios que los estudios aportan a nuestra dicha.

Palabras clave: felicidad, bienestar subjetivo, educación, estudiantes.

\section{HOW MUCH IS YOUR HAPPINESS WORTH}

\section{Abstract}

This article reviews the high level of subjective well-being of Mexicans and especially of Mexican students. To give an account of the high value that studying has in our happiness, I try to give a monetary expression to the tenths that add up our average of subjective well-being. Likewise, I conclude with some considerations regarding certain aspects that could further promote the benefits that studying brings to our happiness.

Key words: happiness, subjective well-being, education, students. 


\section{Gonzalo Soltero}

gsoltero@enes.unam.mx

Actualmente es profesor titular en la Escuela B. Profesor fundador de la ENES León de la UNAM, autor de cinco libros de narrativa y doctor en estudios de política cultural por la Universidad de Warwick, Gran Bretaña. Forma parte del Sistema Nacional de Investigadores y ha sido miembro del Sistema Nacional de Creadores de Arte en dos ocasiones. Sus áreas de investigación son la política cultural, el bienestar subjetivo, la literatura mexicana contemporánea y la construcción narrativa de problemas sociales.

\section{Introducción}

México es un país contradictorio. Los medios nos bombardean a cada momento con malas noticias, que no carecen de fundamento. Tras una década extremadamente violenta, el año pasado, 2017, superó a los anteriores como el año con más homicidios desde que se tiene registro (Secretariado Ejecutivo del Sistema Nacional de Seguridad Pública, 2018). A la vez, 43.6 \% de la población (más de 55 millones de personas) vive en condiciones de pobreza (Consejo Nacional de Evaluación de la Política de Desarrollo Social (CONEVAL), 2017). No es raro escuchar comentarios sobre la descomposición del país, que somos un Estado fallido o que no tenemos futuro. Y a pesar de lo anterior, según encuestas nacionales e internacionales somos uno de los países más felices del mundo. El segmento más feliz de los adultos mexicanos tiene de 18 a 29 años de edad y la actividad que mayor satisfacción de vida produce es la de ser estudiante (Instituto Nacional de Estadística y Geografía (INEGI), 2016b, 2017a).

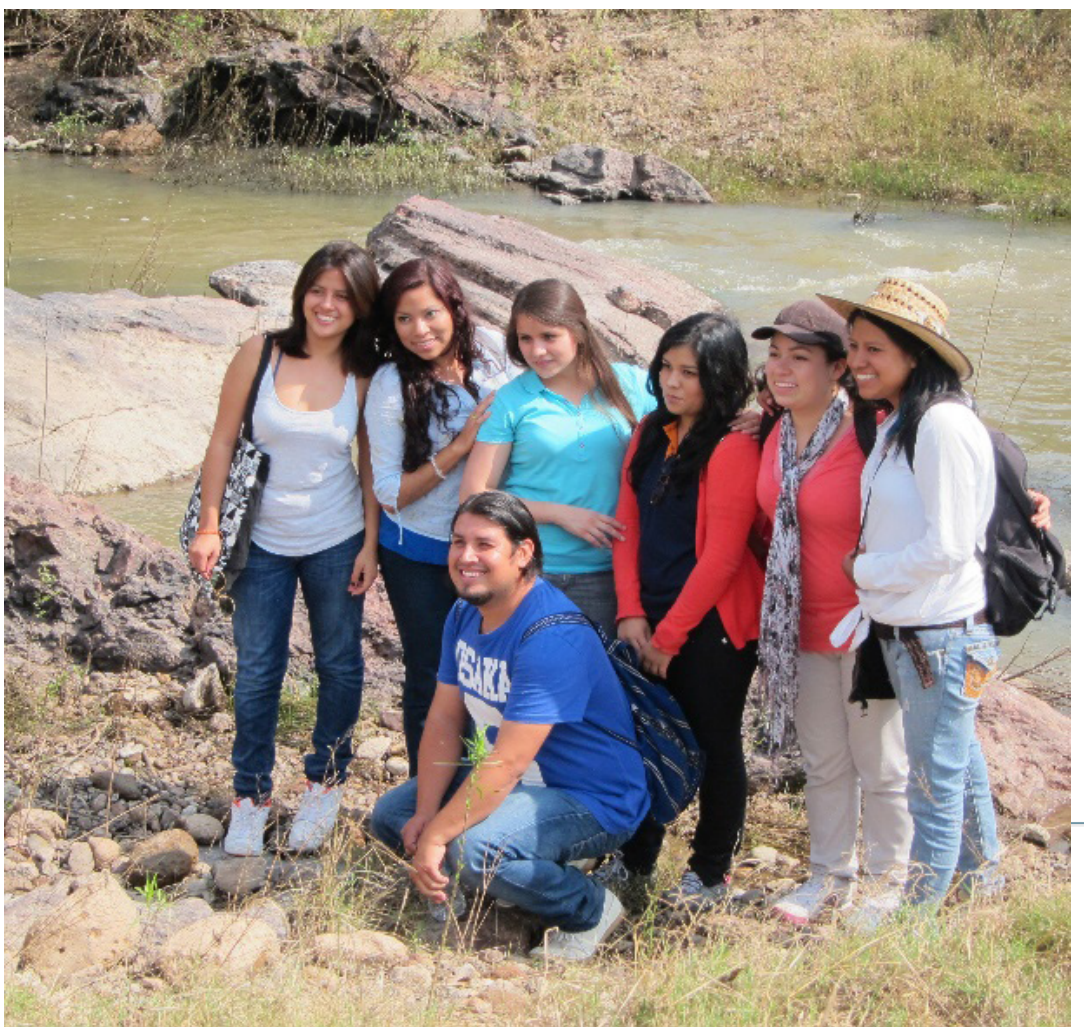

Desde hace algunos años, el bienestar subjetivo, entendido por lo general como la suma de felicidad y satisfacción de vida, comenzó a ser medido como un aspecto del desarroIlo. En México los primeros resultados a cargo del INEGI salieron en 2012 y aseguraban que la mayoría de los mexicanos somos felices. Esta conclusión provocó revuelo en los medios de comunicación por el contraste ante lo que ellos consignan diariamente sobre nuestra debacle nacional. No sólo están las mediciones del INEGI, pues otras encuestas, nacionales y extranjeras, coinciden con esta paradójica felicidad mexicana (por ejemplo, ENSAVISO, 2015; Flores, 2015; INEGI, 2012, 2017a; Pew Research Center, 2014; New Economics Foundation, 2016). 


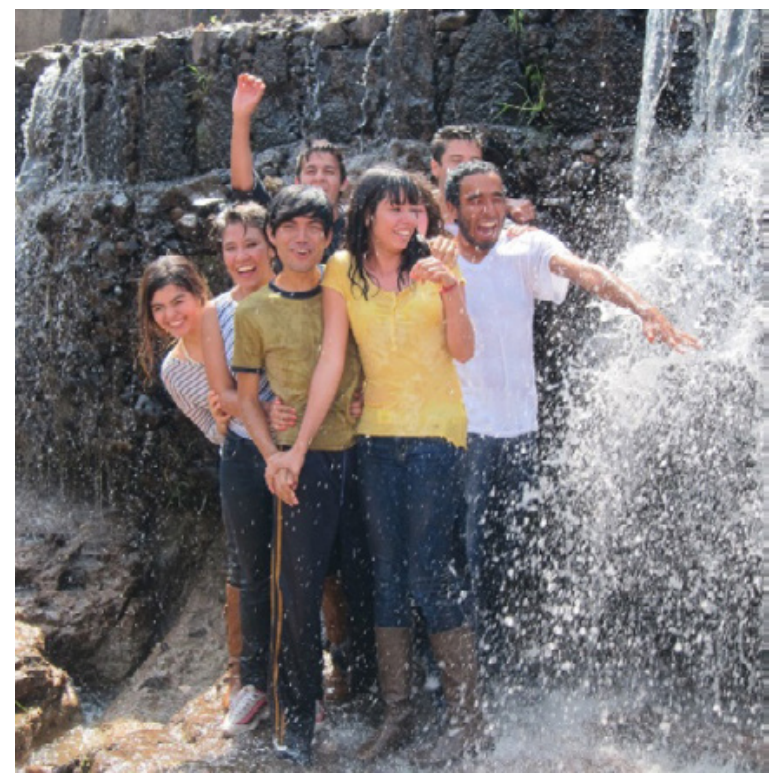

¿Se puede medir la felicidad?

Pero demos un paso atrás, ¿es posible medir la felicidad? ¿Cómo, si la definición que cada uno tiene puede ser muy diferente a la que tienen otros, incluso entre nuestros amigos y familiares? La idea principal es que, si cada quien tiene su propia definición de felicidad, también cada quien sabe qué tanto se acerca a ella, o no. Por eso la mejor fuente para profundizar en el bienestar subjetivo son los propios sujetos: ¿qué piensan y sienten sobre su propia vida? Las mediciones consisten en pedirles que califiquen en una escala del 1 al 10 diversos aspectos relacionados con su felicidad y satisfacción; de acuerdo con ellas nuestro promedio nacional está por encima de 8. Nuestra principal fuente de felicidad y satisfacción son las relaciones personales, especialmente la familia, mientras que, en concordancia con los problemas mencionados en el primer párrafo, la mayor insatisfacción la causa la situación del país, sobre todo la inseguridad ciudadana (INEGI, 2017a).

\section{¿Qué nos da felicidad en México?}

Ahora, ¿qué nos resta o qué nos da mayor dicha, y qué tanto? Comencemos por la sociabilidad, que es la manera de relacionarnos con los demás, y el principal pilar de la felicidad en México. Estar casado es el estado conyugal que más bienestar subjetivo trae a la población, de una a cuatro décimas por encima de los divorciados, viudos, separados o quienes viven en unión libre. Reunirse con familiares o amigos en los 30 días previos a la encuesta trae una diferencia de 1/2 punto a no haberse reunido con ellos; este incremento se mantiene aún si el contacto es mediante teléfono o algún medio digital. También se incluye la sociabilidad hacia las mascotas y, contrario a los que muchos pensarían, en promedio éstas bajan una décima de satisfacción de vida; y un gato baja dos décimas más que tener un perro u otro tipo de animal (INEGI, 2016a).

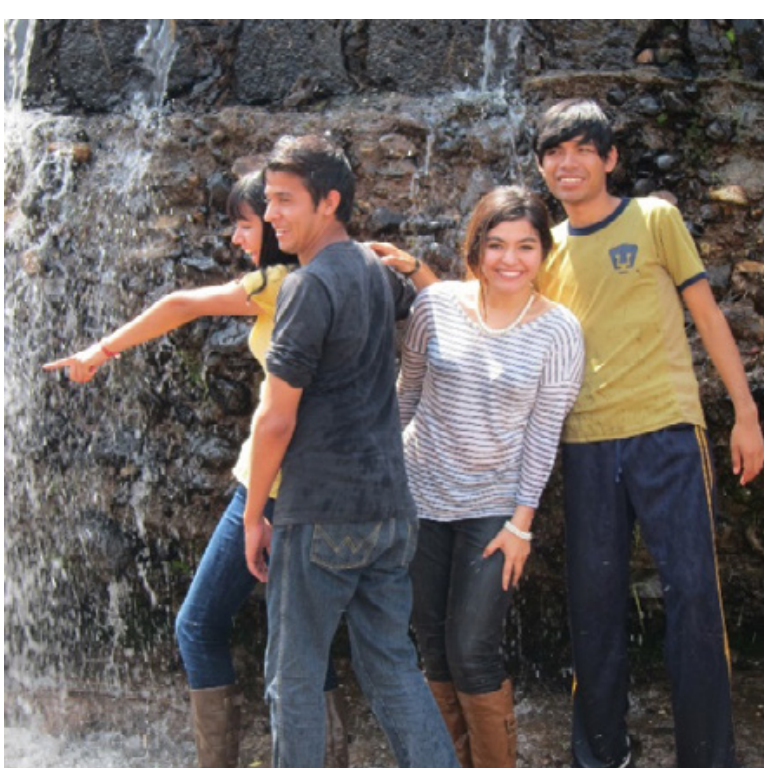


Uno de los factores que más merma la satisfacción de vida mexicana es la discriminación. Dependiendo de nuestro color de piel, etnia, aspecto físico o clase social podemos en promedio perder hasta un punto de felicidad...

Otro aspecto importante son los recursos materiales con que se cuenta. Si algo han comprobado los estudios sobre la felicidad es que el dinero es fundamental para alcanzarla, pero no la compra. Es decir, cuando no se pueden cubrir las necesidades básicas, por ejemplo, atender a un hijo enfermo, se es infeliz. Por ello, conforme aumenta el ingreso aumenta el bienestar subjetivo, pero a partir de que estas necesidades quedan cubiertas la felicidad se estanca, aunque el ingreso siga subiendo. Coleccionar autos deportivos o ganar millones no aumenta la felicidad. En términos de política pública este punto es fundamental pues señala la importancia de buscar mayor igualdad social, contrario a lo que ha sucedido en el mundo en las últimas décadas.

En México, el INEGI ordena de menor a mayor según su nivel de ingreso a todos los hogares del país y los divide en 10 grupos o deciles. Cada grupo contiene a un $10 \%$ de las familias mexicanas. El ingreso promedio en el primer decil es de $\$ 1,110$ pesos mensuales por perceptor, mientras que en el décimo es de \$22,980 (INEGI, 2017b). Entre ganar lo primero y lo segundo hay una diferencia de 1.2 puntos en la felicidad, una distancia considerable, pero a la vez menor de lo que podría suponerse (INEGI, 2016a). Recordemos que la felicidad se mide del 1 al 10, así que supongamos el caso de una persona que pertenece al decil más rico, cuyo bienestar subjetivo fuera 8 (como el promedio nacional): esa misma persona vería su bienestar subjetivo reducido a 6.8 en caso de tener los ingresos del decil más pobre, al restar esas 12 décimas de felicidad.

\section{¿Cuánto vale ser feliz?}

En otros países se le ha asignado una expresión monetaria a la felicidad para entenderla mejor y divulgar entre la población el valor de lo que nos hace felices o no. Por ejemplo, nuestras relaciones personales son tan importantes que un estudio en Gran Bretaña intentó "ponerle precio" a nuestros familiares, amigos y vecinos: una mejoría en nuestra relación con ellos tendría un valor en nuestra satisfacción de vida equivalente a 85 mil libras esterlinas al año, es decir más de 2 millones de pesos (Powdthavee, 2008). Por otro lado, un matrimonio duradero valdría 100 mil dólares por año (Blanchflower y Oswald, 1999). El mismo procedimiento se ha llevado a cabo para calcular la pérdida de un ser querido. Para "compensar" la muerte de una madre se necesitaría 20 mil libras anuales, para la de un hijo 41 mil y para la de un cónyuge 64 mil. Este cálculo parte de estudios que registran qué tanto bienestar subjetivo se pierde ante estos eventos 
y, aunque puedan sonar morbosos o materialistas, su utilidad práctica es considerable, por ejemplo, para calcular la indemnización que le correspondería a una persona en caso de un accidente que como consecuencia tuviera una de las pérdidas mencionadas (Oswald y Powdthavee, 2007).

\section{Ser feliz en México}

En México no se han llevado a cabo cálculos semejantes. Hacerlo con precisión requiere una destreza que me excede, pero intentaré una aproximación. A partir de la relación que hay entre el ingreso económico y el bienestar subjetivo (es

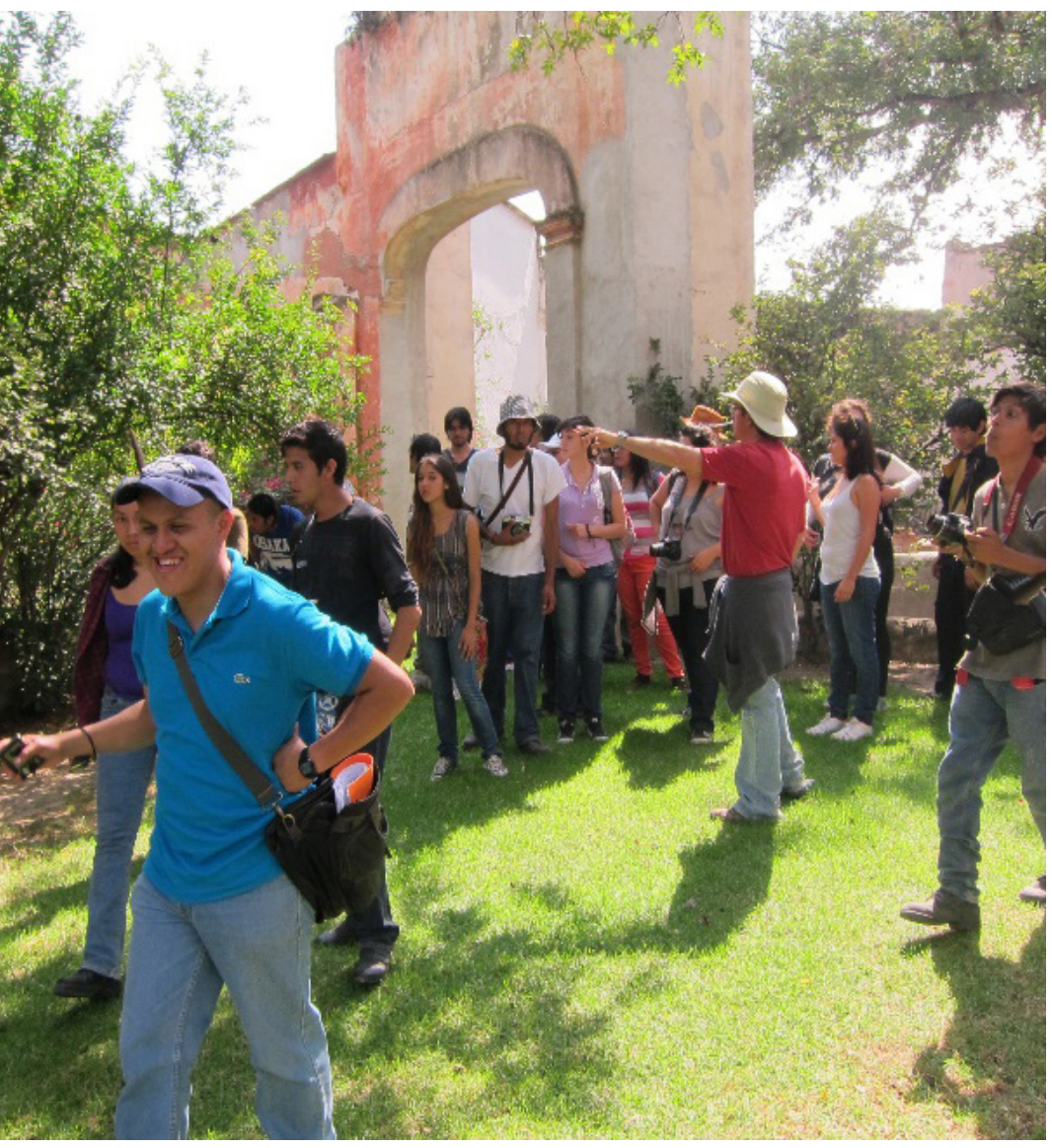
decir, qué tanto afecta nuestra felicidad si ganamos más o menos por mes), podríamos aventurar un primer cálculo. Si dividimos la diferencia de ingreso entre el decil más rico y el más pobre entre las décimas de bienestar subjetivo que dicha diferencia ocasiona, redondearíamos el valor de la décima de felicidad mexicana en 2 mil pesos mensuales. Veamos qué cálculos salen a partir de esto.

Uno de los factores que más merma la satisfacción de vida mexicana es la discriminación. Dependiendo de nuestro color de piel, etnia, aspecto físico o clase social podemos en promedio perder hasta un punto de felicidad (INEGI, 2016a). La pérdida de bienestar subjetivo es semejante por la discriminación ante la preferencia sexual. Nuestro país no es el único. Blanchflower y Oswald (2004) calcularon que para desagraviar a la población negra de Estados Unidos por el racismo que vive en ese país, cada uno debería recibir 30 mil dólares anuales. En un ejercicio semejante, si la décima de felicidad equivale a 2 mil pesos, la discriminación en México tendría un costo de 20 mil pesos mensuales, puesto que reduce la felicidad en una escala de un punto completo. Además, las discriminaciones van sumando su efecto: si soy moreno, obeso, pobre y gay, el efecto negativo de la reacción de los demás hacia estas características juntas reducirá mi felicidad en mayor medida que si sólo tuviera una de ellas. Por ejemplo, el grupo con el bienestar subjetivo más castigado en nuestro país son las mujeres indígenas que no hablan español; al juntar esas tres características, su bienestar subjetivo se ve afectado con 1.6 puntos menos, por lo que, en este caso, su compensación se incrementaría a 32 mil pesos mensuales. 


\section{¿Cuánto vale la felicidad que da la educación en México?}

Pasemos ahora a un tema mucho más luminoso para nuestra felicidad. Como mencioné al principio, los adultos mexicanos más dichosos tienen entre 18 y 29 años de edad. Asimismo, la actividad que mayor satisfacción de vida produce a los mexicanos a partir de los 12 años es la de ser estudiante. A pesar de las quejas que los padres y profesores escuchamos sobre la escuela, el impacto que ésta tiene sobre nuestro bienestar subjetivo es impresionante.

En cuanto a ocupaciones, quienes se encuentran desempleados tienen el nivel más bajo de estar satisfechos o muy satisfechos con la vida (6.4). La distancia entre ellos y quienes tienen un empleo (7.7) es considerable: 1.3 puntos (INEGI, 2016b). Esto permite ilustrar uno de los puntos antes mencionados, pues tener trabajo ocasiona mayor satisfacción que la diferencia entre formar parte del $10 \%$ más pobre y el más rico del país.

Ahora, la felicidad de los estudiantes se encuentra nueve décimas por encima a la de la población laboralmente activa (8.6). Esto quiere decir que su nivel de bienestar sería equivalente a una renta mensual de 18,000 pesos sobre los que tienen un empleo y 44,000 sobre los que no. Es interesante hacer notar que dentro de la misma población estudiantil hay diferencias semejantes.

El mejor posicionamiento en términos de satisfacción con la vida y balance anímico-afectivo lo da haber entrado a la escuela que deseaban (8.27) y le sigue muy de cerca el grupo de quienes se graduaron o concluyeron satisfactoriamente su ciclo escolar (8.20). En el lado contrario, quienes no pudieron entrar

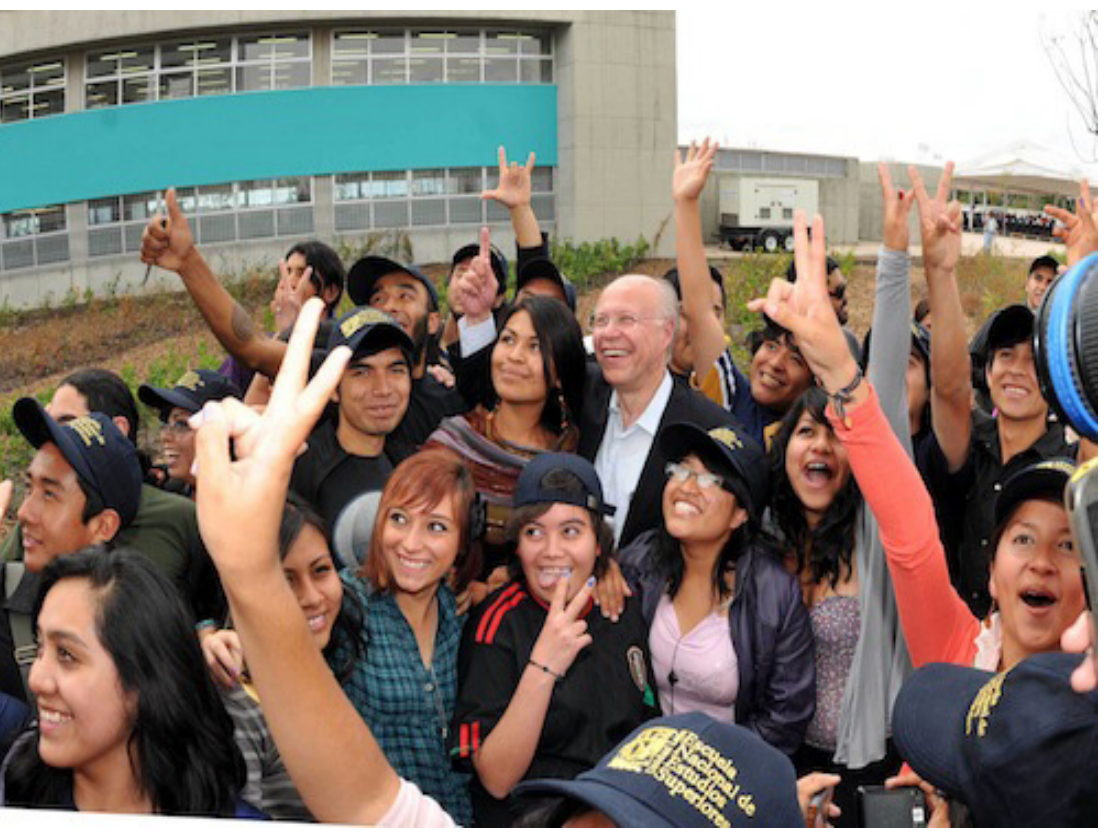
a la escuela de su elección están 4 décimas por abajo (7.8), y aquellos que debieron abandonar sus estudios otras 4 décimas menos (7.4) (INEGI, 2015). Si ponemos como ejemplo a un alumno que logró ingresar a la UNAM, como lo deseaba, pero que tiene que desertar debido a problemas económicos, dicha circunstancia le traería una merma de 8 décimas de bienestar subjetivo, equivalente a 16,000 pesos mensuales, lo cual abona a la justificación de los programas de becas que permiten a los estudiantes seguir siéndolo.

En cada emisión de resultados del Programa Internacional de Evaluación de los Alumnos (PISA) de la Organización para la Cooperación y el Desarrollo Económicos (OCDE) salen a relucir algunas deficiencias de nuestro 
sistema educativo, pues los niños mexicanos tienden a ocupar el último lugar de los 35 países miembros. Recientemente, el informe PISA analizó por primera vez la satisfacción y felicidad de los estudiantes, y aunque México es la nación con el peor desempeño en educación, también es el país de la OCDE, y el segundo entre los países participantes — tras la República Dominicana- donde la mayor proporción de alumnos declararon ser felices (OCDE, 2017a).

Finalmente, la diferencia en el bienestar subjetivo de los mexicanos entre carecer de educación y obtener un posgrado es de 1.1, casi la misma que hay entre pertenecer al decil más pobre o al más rico en México. Esto quiere decir que la diferencia en términos de felicidad entre no tener educación alguna y el posgrado es de 22,000 pesos mensuales. Como resulta claro, la educación es uno de los elementos que más cuenta para la felicidad. Podría pensarse que esto se debe a que la escolaridad actúa como un predictor del nivel socioeconómico que tendrá el individuo; sin embargo, el papel que juega la educación para el bienestar subjetivo es independiente de los ingresos (Blanchflower y Oswald, 2004).

\section{Obstáculos para la felicidad educativa}

A la par del bienestar subjetivo que trae consigo la educación, hay una tendencia que asocia el aprendizaje y la dedicación al conocimiento con el sufrimiento: un camino lleno de obstáculos con los que se debe lidiar para lograr el título o logro en cuestión. Esto también lo constatan algunos estudios sobre bienestar estudiantil: muchos de quienes están inscritos en educación superior en distintos países sufren una cantidad considerable de estrés y ansiedad. Diversos proyectos de investigación han demostrado la presencia constante de agotamiento emocional y problemas psicológicos relacionados con la presión académica y las dificultades financieras que enfrentan los estudiantes (Andrews y Wilding, 2004).

En México hay una cultura que encomia el sufrimiento y el desgaste; existe incluso un verbo informal, "malpasarse", que designa una especie de martirio voluntario por trabajar sin pausas y sin comer. Uno de los pocos encabezados de la OCDE en los que México tiene el primer lugar es la cantidad de horas transcurridas en el sitio de trabajo, superando a países como Japón y Corea (OCDE, 2017b). Esta actitud, que también predomina en algunos ambientes universitarios, termina siendo más una pose que una pauta de rendimiento, pues hay estudios que demuestran cómo después de 50 horas a la semana la productividad decrece (por ejemplo, Pencavel, 2015), mientras que aumenta con gente feliz y descansada (Oswald, Proto y Sgroi, 2015). El argumento es muy claro para promover la noción de placer con relación al proceso de aprendizaje.

Concluyo con algunos datos que aporta Göran Therborn a favor de la educación. En Estados Unidos un hombre blanco de 50 años que haya completado 
su carrera universitaria tendrá seis años más de vida frente a otro que deje la carrera incompleta. Por otra parte, Therborn (2016) comenta que durante los años noventa en Brasil el hijo de una madre con doce años de estudios tenía una posibilidad diez veces mayor de celebrar su primer cumpleaños que el hijo de una madre analfabeta. Aunque se trata de correlaciones, como todas las que figuran en este artículo, también son argumentos que apuntan a que los beneficios de la educación no son sólo cognoscitivos, sino que son un activo para la vida. A diferencia del empleo, que se puede perder (con la consiguiente disminución del bienestar subjetivo), o la pérdida de un ser querido, la educación se mantiene por el resto de nuestros días, independientemente de los altibajos de nuestra fortuna. En este sentido, la educación es uno de los ingredientes más importantes que tenemos para la felicidad humana; sus beneficios resisten mejor los obstáculos del tiempo e incluso las bajas que nos imponen las leyes de la naturaleza.

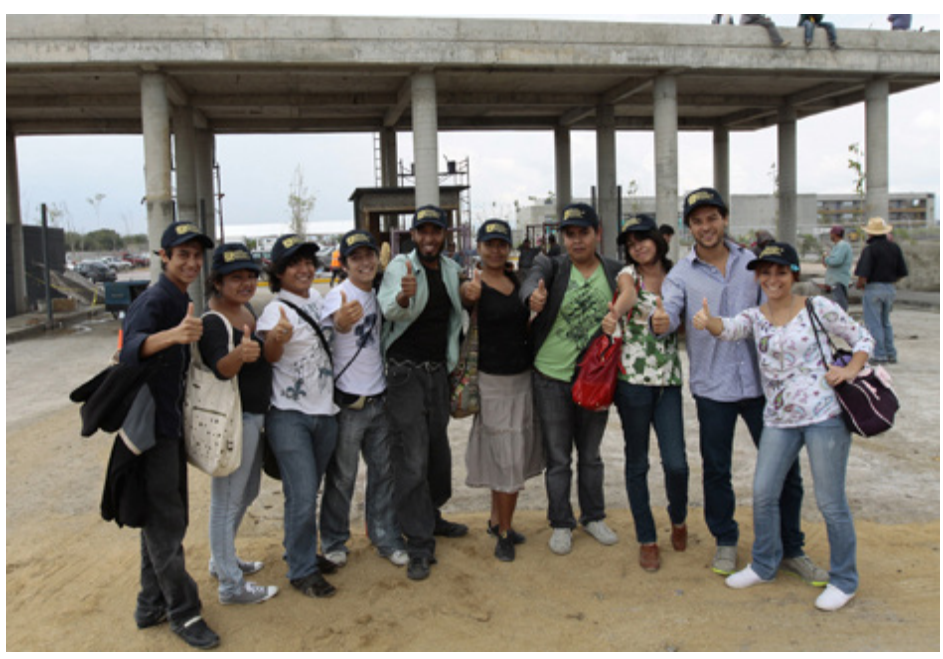

\section{Referencias}

* Andrews, B. y Wilding, J. M. (2004). The relation of depression and anxiety to life stress and achievement in students. British Journal of Psychology, 95(4), 509-521. DOI: https://doi.org/10.1348/0007126042369802

* Blanchflower, D. G. y Oswald, A. J. (2004). Well-Being Over Time in Britain and USA. Journal of Public Economics, 65, 386-396. DOI: https://doi.org/10.3386/w7487

* Consejo Nacional de Evaluación de la Política de Desarrollo Social. (2017, 30 de agosto). CONEVAL Informa la Evolución de la Pobreza 2010-2016 [comunicado de prensa]. Recuperado de: https://www.coneval.org.mx/SalaPrensa/Comunicadosprensa/Paginas/Comunicados-2017.aspx

ENSAVISO-UNAM-IIS. (2015). Encuesta Nacional sobre Satisfacción Subjetiva con la Vida y la Sociedad (ENSAVISO). Ciudad de México: UNAM.

* Flores, J. I. (2015). Sentimientos y Resentimientos de la Nación: Encuesta Nacional de Identidad y Valores. Ciudad de México: UNAM.

* Instituto Nacional de Estadística y Geografía. (2012, 21 de noviembre). Presenta el INEGI Cifras Sobre el Bienestar Subjetivo de Los Mexicanos [boletín de prensa núm. 
431/12]. Recuperado de: https://issuu.com/politicaspublicas/docs/mexicanosybienestar

* Instituto Nacional de Estadística y Geografía. (2015, 20 de octubre). Indicadores de Bienestar Subjetivo de la Población Adulta en México [boletín de prensa núm. 412/15] Recuperado de: http://www.inegi.org.mx/saladeprensa/boletines/2015/ especiales/especiales2015 10 7.pdf

* Instituto Nacional de Estadística y Geografía. (2016a). Uso del Tiempo Libre Módulo Biare Ampliado: Promedios/Satisfacción de Vida. Recuperado de: http://www.beta. inegi.org.mx/proyectos/investigacion/bienestar/ampliado/

* Instituto Nacional de Estadística y Geografía. (2016b). Uso del tiempo y bienestar subjetivo. La Encuesta Nacional sobre Uso del Tiempo (ENUT) 2014. Recuperado de: http://www.beta.inegi.org.mx/proyectos/investigacion/bienestar/tiempo/

- Instituto Nacional de Estadística y Geografía. (2017a). Indicadores de Bienestar Autorreportado de la Población Urbana. Cifras al mes de julio de 2017 [boletín de prensa núm. 391/17]. Recuperado de: http://www.inegi.org.mx/saladeprensa/boletines/2017/biare/biare2017 08.pdf

- Instituto Nacional de Estadística y Geografía. (2017b). Presenta INEGI los resultados de una nueva serie de la encuesta nacional de ingresos y gastos de los hogares (ENIGH) 2016 [boletín de prensa núm. 392/17]. Recuperado de: http://www.inegi.org.mx/ saladeprensa/boletines/2017/enigh/enigh 08.pdf

- New Economics Foundation. (2016). Mexico. Happy Planet Index. Recuperado de: http://happyplanetindex.org/countries/mexico

* Organización para la Cooperación y el Desarrollo Económico. (2017a). PISA 2015 Results (Volume III): Students' Well-Being. París: OECD Publishing. Recuperado de: https://doi.org/10.1787/9789264273856-en

* Organización para la Cooperación y el Desarrollo Económico. (2017b). OECD Employment Outlook 2017. París: OECD Publishing. Recuperado de: http://dx.doi. org/10.1787/empl outlook-2017-en

- Oswald, A. J. y Powdthavee, N. (2007). Death, happiness and the calculation of compensatory damages. Working Paper. Warwick economic research papers [núm. 827]. Coventry: University of Warwick, Department of Economics. Recuperado de: $\quad$ https://warwick.ac.uk/fac/soc/economics/research/workingpapers/2008/ twerp 827.pdf

- Oswald, A. J., Proto, E., y Sgroi, D. (2015). Happiness and Productivity. Journal of Labor Economics, 33(4), 789-822. DOI: https://doi.org/10.1086/681096

* Pencavel, J. (2015). The Productivity of Working Hours. The Economic Journal, 125, 2052-2076. DOI: https://doi.org/10.1111/ecoj.12166

- Pew Research Center. (2014). People in Emerging Markets Catch Up to Advanced Economies in Life Satisfaction. Recuperado de: http://www.pewglobal.org/2014/10/30/ people-in-emerging-markets-catch-up-to-advanced-economies-in-lifesatisfaction/\#richer-publics-more-satisfied-with-life 
* Powdthavee, N. (2008). Putting a price tag on friends, relatives, and neighbours: Using surveys of life satisfaction to value social relationships. Journal of Behavioral and Experimental Economics, 37(4), 1459-1480. DOI: https://doi.org/10.1016/j. socec.2007.04.004

- Secretariado Ejecutivo del Sistema Nacional de Seguridad Pública. (2018). Cifras de homicidio doloso, secuestro, extorsión y robo de vehículos 1997-2017. Recuperado de: http://secretariadoejecutivo.gob.mx/docs/pdfs/cifras\%20de\%20homicidio\%20doloso\%20secuestro\%20etc/HDSECEXTRV 052017.pdf

* Therborn, Göran. (2016). Los campos de exterminio de la desigualdad. México: FCE.

\section{Cómo citar este artículo}

* Soltero, Gonzalo (2018). ¿Cuánto vale tu felicidad?. Revista Digital Universitaria (RDU). Vol. 19, núm. 4 julio-agosto. DOI: http://doi.org/10.22201/codeic.16076079e.2018. v19n4.a7. 